\title{
Optimization of Transit Scheduling Combined with Short-Turn Service Based on Real-Time Passenger Flow
}

\author{
Ailing HUANG ${ }^{\mathrm{a}}$, Yijing MIAO ${ }^{\mathrm{a}}$ and Jiarui $\mathrm{LI}^{\mathrm{a}, \mathrm{b}, 1}$ \\ ${ }^{a}$ Key Laboratory of comprehensive transportation big data application technology, \\ Beijing Jiaotong University, Beijing 100044, P.R.China \\ ${ }^{\mathrm{b}}$ STV Inc, Los Angeles, CA 90017,U.S.A.
}

\begin{abstract}
In view of a series of problems, such as unable to meet the needs of passengers, high full load ratio or waste of carrying capacity on unbalanced passenger flow sections caused by the all-stop fleet scheduling in the urban public transit system, this paper proposed a bus combination scheduling strategy with considering short-turn service based on the imbalance coefficient of passenger flow and a method to determine the turning back point. A combined dispatching optimization model is established with the objective function of minimizing the total system cost which includes the waiting time cost of passengers, the congestion feeling cost and the operation cost of public transit enterprises. The headways of short-turn and all-stop scheme are optimized by the combined scheduling model, and the solution method is proposed. Taking Beijing No. A bus line as an empirical analysis object, the real-time passenger flow and vehicle data in a working day are collected and analyzed, and the optimized scheme of shortturn service combination scheduling is obtained. The results show that compared with the traditional all-stop fleet scheduling, the optimized short-turn service combination scheduling can reduce the fleet size by $4.9 \%$ and effectively improve the operation efficiency and system benefits.
\end{abstract}

Keywords. Public transit; combined scheduling; short-turn service; total system cost; operation

\section{Introduction}

The problems related to public transit operation are the focus and difficulty of public transit research. At present, the bus dispatching in major cities in China is still based on all-stop fleet scheduling, which often leads to a surge in passenger flow and traffic congestion during peak hours. Therefore, it is an important topic to explore the rationalization of urban public transit routes and vehicle scheduling optimization.

At present, the research on all-stop fleet scheduling has been very in-depth. Cancela summarized the international representative research work on bus dispatching and network design [1]. The traditional bus dispatching research focuses on dealing with the unbalanced spatial and temporal distribution of passenger flow, with the purpose of saving passenger travel cost [2-4]. The main research directions include bus timetable formulation [5-7], departure frequency optimization [8-10], routes scheduling

\footnotetext{
${ }^{1}$ Jiarui LI, 1055 W 7 $7^{\text {th }}$ St \#3150, Los Angeles, CA 90017; E-mail: jiarui.li@stvinc.com
} 
optimization [11-12]. In recent years, many achievements have been made in bus combination scheduling design. Among them, short-turn service is considered as a feasible way to reduce the cost, and due to the limited number of research, it is worth further study.

In order to adapt to the travel demand of the section with the highest passenger load and prevent overloading, Canca et al. [13] proposed a model to increase the departure frequency of certain stops on the line and balance the vehicle occupancy level. The setting of turning back point should shorten the passenger travel time on the premise of ensuring the service quality. Cheng [14] used the "cutting method" to determine the stops of short-turn bus lines, and established the optimization model of short-turn service combination scheduling with the optimization objectives of passenger waiting cost, congestion cost and bus operation cost under different scheduling modes. In this proposed model, the all-stop and short-turn service depart in a certain proportion, and different proportions represent different departure modes. Cortés et al. [15] established an optimal scheduling model for short-turn service on single line. It is proved that the total cost can be reduced by reducing the extra operating cost of empty vehicles. According to the theory of deficit function, in the process of combined dispatching of all-stop and short-turn service, $\mathrm{Hu}$ et al. [16] realized all departures of single line with the least number of vehicles by properly inserting idle vehicles, making full use of idle vehicles and reducing the operation cost of public transit group. Based on the passenger flow data, Cao [17] took the passenger waiting and riding satisfaction and the bus company satisfaction as the objective function, used the power weighted sum method to process the multi-objective function, established a single objective public transport scheduling model, and used the improved genetic algorithm to solve the problem. Chen et al. [18] constructed a combined scheduling model under the conditions of limited vehicle capacity and random passenger travel, and verified the model and solution method through an example. Wei et al. [19] put forward a kind of multi-objective single line combined dispatching method for stop-skipping service, and discussed the relationship among departure frequency, turning back point and cost with an example. Canca et al. [20] proposed a short-turn service scheduling strategy under the premise of ensuring certain service quality to reduce waiting time of passengers. Zhang et al. [21] established a mixed scheduling model of stop-skipped and short-turn service based on real-time data. Compared with the traditional scheduling mode, the hybrid scheduling strategies can effectively reduce the total cost of public transit system. On the basis of considering the number of passengers waiting to be transported due to the limitation of vehicle capacity and the dwelling time, Gao [22] set up the short-turn service combination scheduling model with the weighted sum of passenger travel cost and bus enterprise cost as the objective function, where the scheduling mode and departure interval as the decision variables. Ming et al. [23] discussed the combination of different departure modes and intervals, established a combination bus scheduling model with uncertain departure frequency, then compared and proved that multi-mode combined bus dispatching can effectively reduce the system time cost. On this basis, Liu [24] proposed a hybrid heuristic algorithm combining particle swarm optimization algorithm and pattern search algorithm to solve the problem of combination scheduling model with different departure frequencies. Luo [25] established an all-stop combined dispatching model with the objective function of minimizing the operating cost of passengers and bus companies by adopting appropriate departure strategy of vehicle groups to optimize the 
original departure interval and adjust the driving plan to realize comparatively even distribution of passenger flow.

Taken together, the existing research, especially the practical application on the mode of combined bus dispatching is still in its infancy. Although more research of bus scheduling optimization has been conducted for many years, several questions remain open in the study of combined scheduling optimization with short-turn service. Since nowadays the real-time data like passenger flow is available via collecting smart card records, which has higher accuracy and reliability, the method of determining shortturn service point needs to be further explored. It is more reasonable to use the realtime passenger flow data and the actual traffic conditions as the data source of model input to judge the turning back point of short-turn service. It means that a systematic framework of designing transit combination scheduling scheme based on the real-time data needs to design. In addition, most of the studies only chooses one of the user cost and operation cost as the objective function, and accordingly, the optimized results cannot reflect the benefits and effectiveness more completely. Moreover, the cost in different scheduling schemes also need to be studied in detail. Therefore, the systematic objective needs to be optimized further with considering both the benefits of users and operators, and the benefit of optimized scheme is worth to be discussed more comprehensively. All of these factors should be thoroughly considered in the modeling framework. To address the research limitations that are discussed above, this paper designs a framework to judge the short-turn point based on the real-time passenger flow, and proposes a model and solution algorithm to optimize the bus combination scheduling scheme. In our model, different scheduling strategies combined with allstop and short-turn services are designed and the minimum sum of the cost of both sides are regarded as the optimization goal from the perspectives of passengers and public transit enterprises. Particularly, besides the waiting cost, the congestion feeling cost of passengers is taken into account in our modeling framework to reflect more real external cost. Moreover, to evaluate the feasibility of the proposed model and algorithm, this paper conducts a case study of a bus line in Beijing by using the real data and makes an analysis on the optimized results. The results shows that our proposed method can greatly reduce the cost of passengers and operator, and improve the operational efficiency. The main purpose of this paper is to optimize the bus scheduling by formulating a feasible and effective combined dispatching strategy, reduce the passenger cost and the operation cost, and evacuate the passenger flow in peak period immediately and effectively. The proposed method can be applied to the operation and scheduling practice by public transit enterprises in the future.

The remainder of this paper is organized as follows. The second part gives the description of research object, and the judgement and planning framework of short-turn service combination dispatching mode. In the third part, based on the previous steps, the bus combination schedule model is proposed and the solving method is given. A case study is conducted in the fourth section to illustrate the specific application of the proposed methodology, where a satisfactory results comparison is discussed. The last section concludes this paper and suggests feasible directions for future study. 


\section{Framework of bus combination scheduling design with real-time passenger flow}

\subsection{Bus dispatching mode}

Vehicle scheduling is a variety of methods adopted in the preparation of public transit operation plan. According to the passenger flow distribution, the combined bus dispatching is a bus dispatching mode supplemented by other dispatching forms on the basis of all-stop service.

- All-stop service

The all-stop service refers to the scheduling form in which the bus stops in sequence at the passing stop, does not skip stops in the middle, and finally stops at the terminal.

- Short-turn service

Short-turn service is a kind of scheduling mode that stops at specific stops or sections with large passenger flow. It stops at each stop in the operation section. According to the setting of initial and terminal stops, it can be divided into three forms: midway turning back, turning back from midway stop to another midway stop, and turning back from midway stop to terminal stop.

\subsection{Judgment of combination dispatching mode with considering short-turn service}

Based on the unbalanced coefficient, the judgment method of short-turn service combination dispatching mode is constructed. It can be expressed by the road section unbalanced coefficient $K_{s i}$ or section passenger flow difference $\Delta Q_{i}$, as shown in Eq. (1) and (2)

$$
\begin{gathered}
K_{s i}=\frac{Q_{s i}}{\overline{Q_{n}}} \\
\Delta Q_{i}=Q_{s i}-\overline{Q_{n}}
\end{gathered}
$$

In Eq. (1) and (2), $Q_{s i}, \overline{Q_{n}}$ respectively refer to the passenger flow of road section $i$ and the average section passenger flow within the statistical time.

When the passenger flow is high and the unbalanced coefficient of road section $K_{s i}>1.2$, it can be considered to carry out short-turn service combination scheduling in the section, so as to reduce the congestion of passenger flow in peak road section. The specific determination steps are as follow:

- Step 1: Make statistics on the passenger flow of to be investigated, and calculate the road section unbalance coefficients of the upward and downward directions respectively;

- Step 2: According to the criteria of short-turn service, determine the stop which will operate the short-turn service. Table 1 is the judgment standard of the short-turn service; 
Table 1. Criteria for judging short-turn service

\begin{tabular}{lll}
\hline \multicolumn{1}{c}{ Judgment index } & Condition & \multicolumn{1}{c}{ Remarks } \\
\hline $\begin{array}{l}\text { Unbalanced coefficient of road } \\
\text { section }\end{array}$ & $K_{s i}>K_{s}^{0}$ & Generally, $K_{s}^{0}=1.2 \sim 1.5$ \\
$\begin{array}{l}\text { Section passenger flow } \\
\text { difference }\end{array}$ & $\Delta Q_{i} \geq \eta q_{0}$ & $\begin{array}{l}\text { Generally, } \eta=2 \sim 4, q_{0} \text { is the rated } \\
\text { capacity of the vehicle }\end{array}$ \\
\hline
\end{tabular}

- Step 3: To evaluate and investigate the traffic condition of routes and sections where short-turn service will be set up to judge whether the selected stop is feasible;

- $\quad$ Step 4: Test the vehicles used in the section to determine the vehicle types and total fleet size of the bus line;

- Step 5: Formulate the departure interval and timetable for the all-stop and short-turn service.

In addition to using the unbalanced coefficient method to determine the short-turn service dispatching mode, it is also necessary to consider the actual road traffic conditions, the scale of public transit enterprises, the economic level of urban residents and other factors, so as to formulate a dispatching scheme which is beneficial to both passengers and public transit enterprises.

\subsection{Design process of combination scheduling scheme based on real-time flow}

When the short-turn service combination is determined, the combined scheduling model of all-stop and short-turn service is established. The real-time passenger flow data and vehicle data is input into the model. Through solution, the optimized departure interval is obtained, and the corresponding departure schedule and driving scheme are formulated. The specific process is shown in Figure 1.

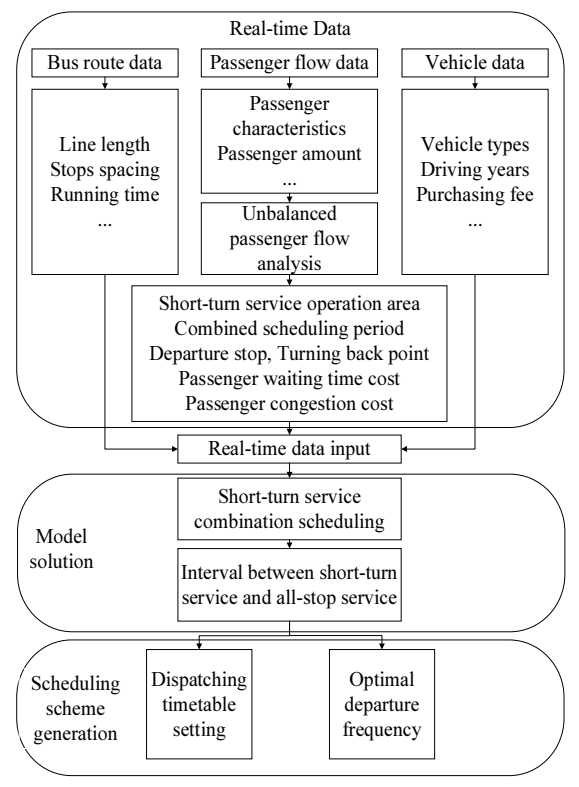

Figure 1. Flow chart of combined scheduling 


\section{Bus combination schedule modeling and solution}

\subsection{Model establishment}

\subsubsection{Assumptions}

- The principle of bus passengers is that the passengers who arrive at bus stop first have priority and obey the uniform distribution;

- The types of bus vehicles are consistent, and there are no multiple types;

- The fixed cost of public transit enterprises is related to the fleet size of bus line;

- The variable cost of public transit enterprises is related to vehicle fuel consumption;

- There is no transfer between all-stop service and short-turn service;

- In the combined dispatching, the difference of stopping time between the allstop service and short-turn service is not considered.

\subsubsection{Parameters}

The model parameter definition of the short-turn service combination scheduling model is shown in Table 2.

Table 2. Model variable table

\begin{tabular}{cll}
\hline $\begin{array}{c}\text { Parameter } \\
\text { symbol }\end{array}$ & \multicolumn{1}{c}{ Meaning } & Unit \\
\hline $\boldsymbol{l}$ & Stop serial number & - \\
\hline$\mu$ & Sequence of short-turn service departure stop & - \\
\hline & Turning back point sequence of short-turn service & - \\
\hline & $\begin{array}{l}\text { Parameters of combined dispatching mode, } \\
m=0,1,2 ; \text { where 0 is the all-stop scheduling, 1 is } \\
\text { the combined scheduling of one all-stop service and } \\
\text { short-turn combination, and 2 is two all-stop service } \\
\text { and short-turn combination scheduling }\end{array}$ & - \\
\hline$l_{i}$ & Parameters of congestion penalty cost coefficient & - \\
\hline$L$ & Stop spacing & $\mathrm{km}$ \\
\hline$L_{s}$ & Total length of bus line & $\mathrm{km}$ \\
\hline$\tau$ & Study duration & $\mathrm{km}$ \\
\hline$h_{0}$ & Departure interval under whole vehicle scheduling & $\mathrm{min}$ \\
\hline$h_{1}$ & $\begin{array}{l}\text { Time interval of the next short-turn service after an } \\
\text { all-stop service }\end{array}$ & $\mathrm{min}$ \\
\hline$h_{2}$ & $\begin{array}{l}\text { Time interval of the next all-stop service after a } \\
\text { short-turn service }\end{array}$ & min \\
\hline
\end{tabular}




\begin{tabular}{|c|c|c|}
\hline$f$ & Total departures & second \\
\hline$r_{i k}$ & $\begin{array}{l}\text { Arrival rate of passengers getting on at stop } i \text { and } \\
\text { off at stop } k\end{array}$ & person $/ \mathrm{min}$ \\
\hline$P_{i}^{(m)}$ & $\begin{array}{l}\text { Under mode } m \text {, the number of boarding passengers } \\
\text { at stop } i\end{array}$ & person \\
\hline$Q_{i}^{(m)}$ & $\begin{array}{l}\text { Under mode } m \text {, the number of alighting passengers } \\
\text { at stop } i\end{array}$ & person \\
\hline$N_{i}^{(m)}$ & $\begin{array}{l}\text { Under mode } m \text {, the cumulative number of } \\
\text { passengers between stop } i \text { and } i+1\end{array}$ & person \\
\hline$W_{i}^{(m)}$ & $\begin{array}{l}\text { Under mode } m \text {, the waiting time cost of passengers } \\
\text { at stop } i\end{array}$ & yuan \\
\hline$Y_{i}^{(m)}$ & $\begin{array}{l}\text { Under mode } m \text {, when the full load ratio exceeds } \\
\text { maximum load rate, the congestion feeling cost of } \\
\text { passengers from stop } i \text { to } i+1\end{array}$ & yuan \\
\hline$\rho_{i}^{(m)}$ & $\begin{array}{l}\text { Under mode } m \text {, and the congestion penalty cost } \\
\text { coefficient between stop } i \text { and } i+1\end{array}$ & yuan/person $\cdot \mathrm{km}$ \\
\hline$b$ & Rated capacity & person/vehicle \\
\hline$A_{i}^{(m)}$ & Full load ratio of vehicle at stop $i$ & - \\
\hline$A_{0}$ & Critical load ratio & - \\
\hline$\alpha$ & Passenger's waiting time cost & $\begin{array}{l}\text { yuan/person· } \\
\min \end{array}$ \\
\hline$\beta$ & fuel consumption of one vehicle & yuan $/ \mathrm{km}$ \\
\hline$T$ & $\begin{array}{l}\text { Average one-way travel time of all-stop service in } \\
\text { peak hours }\end{array}$ & $\min$ \\
\hline$T_{\mathrm{s}}$ & $\begin{array}{l}\text { Average one-way travel time of short-turn service in } \\
\text { peak hours }\end{array}$ & $\min$ \\
\hline$\xi$ & Bus purchase cost & yuan/vehicle \\
\hline$c$ & Number of drivers per bus & people \\
\hline$d$ & Number of bus seats & individual \\
\hline$R_{0}$ & Driver's daily wage & yuan/person· day \\
\hline$S_{0}$ & Daily vehicle maintenance fee & $\begin{array}{l}\text { yuan/vehicle. } \\
\text { day }\end{array}$ \\
\hline$\theta$ & Permitted driving years of vehicles & year \\
\hline$u_{\min }$ & Minimum fleet size & vehicle \\
\hline
\end{tabular}

\subsubsection{Objective function}

In order to ensure the interests of both passengers and public transit enterprises, the objective function of the short-turn service combination scheduling model constructed 
in this paper includes two parts: passenger cost and operation cost of public transit enterprises.

(1) Passenger cost

The cost of passengers is the sum of waiting cost and congestion cost. The time cost in the bus is not considered because it is not related to bus departure mode. The following is the analysis of different departure conditions.

1) When $m=0$, that is, the bus line only takes the all-stop, the cost of passengers is defined as:

$$
C^{(0)}=\sum_{i=1}^{n-1}\left(W_{i}^{(0)}+Y_{i}^{(0)}\right)
$$

In Eq. (3), the calculation of $W_{i}^{(0)}, Y_{i}^{(0)}$ is as follow:

$$
\left\{\begin{array}{l}
W_{i}^{(0)}=\frac{1}{2} \alpha \sum_{k=i+1}^{n} r_{i k} h_{0}^{2} \\
Y_{i}^{(0)}=\rho_{i}^{(0)}\left(N_{i}^{(0)}-d\right) l_{i}, \quad i=1,2,3, \ldots n-1
\end{array}\right.
$$

Where, $N_{i}^{(0)}$ is as shown in Eq. (5); $\rho_{i}^{0}$ is as shown in Eq. (6).

$$
N_{i}^{0}=N_{i-1}^{0}+P_{i}^{0}-Q_{i}^{0}=N_{i-1}^{(0)}+\sum_{k=i+1}^{n} r_{i k} h_{0}-\sum_{l=1}^{i-1} r_{l i} h_{0}
$$

In Eq. (5), $Q_{1}^{(0)}=0, N_{1}^{(0)}=P_{1}^{(0)}$.

$$
\rho_{i}^{(0)}=\left\{\begin{array}{l}
\varepsilon A_{i}^{0}, 0 \leq \varepsilon \leq 1, A_{i}^{0} \geq A_{0} \\
0, A_{i}^{0}<A_{0}
\end{array}\right.
$$

In Eq. (6), the calculation of $A_{i}^{(0)}$ is as follow:

$$
A_{i}^{(0)}=\frac{N_{i}^{(0)}}{b}
$$

2) When $m=1$, a combined dispatching of one all-stop service and the short-turn service is issued. The stop sequence of short-turn service is $\mu, \mu+1, \cdots, \varphi$, then the passenger cost of short-turn service is defined as:

$$
C^{(1)}=\sum_{i=\mu}^{\varphi-1}\left(W_{i}^{(1)}+Y_{i}^{(1)}\right)
$$

In Eq. (8), the calculation of $W_{i}^{(1)}, Y_{i}^{(1)}$ is as follow:

$$
\left\{\begin{array}{l}
W_{i}^{(1)}=\frac{1}{2} \alpha \sum_{k=i+1}^{\varphi} r_{i k} h_{1}^{2} \\
Y_{i}^{(1)}=\rho^{(1)}\left(N_{i}^{(1)}-d\right) l_{i}, \quad i=\mu, \mu+1, \ldots \varphi-1
\end{array}\right.
$$

In Eq. (9), $N_{i}^{(1)}$ can be calculated by Eq. (10), and $\rho_{i}^{(1)}$ can be calculated by Eq. (11). 


$$
N_{i}^{(1)}=N_{i-1}^{(1)}+P_{i}^{(1)}-Q_{i}^{(1)}=N_{i-1}^{(1)}+\sum_{k=i+1}^{\varphi} r_{i k} h_{1}-\sum_{l=\mu}^{i-1} r_{l i} h_{1}
$$

In Eq. (10), there is $Q_{\mu}^{(1)}=0, N_{\mu}^{(1)}=P_{\mu}^{(1)}, i=\mu, \mu+1, \ldots \varphi-1$.

$$
\rho_{i}^{(1)}=\left\{\begin{array}{l}
\varepsilon A_{i}^{(1)}, 0 \leq \varepsilon \leq 1, A_{i}^{(1)} \geq A_{0} \\
0, A_{i}^{(1)}<A_{0}
\end{array}\right.
$$

In Eq. (11), the calculation of $A_{i}^{(1)}$ is as follow:

$$
A_{i}^{(1)}=\frac{N_{i}^{(1)}}{b}
$$

3) When $m=2$, the passenger cost of the all-stop scheduling is defined as:

$$
C^{(2)}=\sum_{i=1}^{n-1}\left(W_{i}^{(2)}+Y_{i}^{(2)}\right)=\sum_{i=1}^{\mu-1} W_{i}^{(2)}+\sum_{i=\mu}^{\varphi-1} W_{i}^{(2)}+\sum_{i=\varphi}^{n-1} W_{i}^{(2)}+\sum_{i=1}^{n-1} Y_{i}^{(2)}
$$

In Eq. (13), the location relationship between stop $i$ and the range of short-turn service section should be considered when calculating $W_{i}^{(2)}$, which is discussed in the following different cases.

a) When $1 \leq i \leq \mu-1$ or $\varphi \leq i \leq n-1$, stop $i$ is not within the scope of shortturn service section, passengers need to wait for the next all-stop service:

During period $h_{1}+h_{2}$, the sum of passengers boarding at stop $i$, which is denoted as $P_{i}^{(2)}$, can be expressed as

$$
P_{i}^{(2)}=\sum_{k=i+1}^{n} r_{i k}\left(h_{1}+h_{2}\right), 1 \leq i \leq \mu-1 \text { or } \varphi \leq i \leq n-1
$$

Then the average waiting time of passengers is $\frac{1}{2}\left(h_{1}+h_{2}\right)$, and the calculation of passengers waiting time cost $W_{i}^{(2)}$ is obtained as follow:

$$
W_{i}^{(2)}=\frac{1}{2} \alpha \sum_{k=i+1}^{n} r_{i k}\left(h_{1}+h_{2}\right)^{2}
$$

b) When $\mu \leq i \leq \varphi-1$, that is, stop $i$ is within the range of short-turn service section:

At this time, the number of passengers who do not take short-turn service bus because they cannot reach their destination directly is $\sum_{k=\varphi+1}^{n} r_{i k} h_{1}$, and the waiting time cost of these passengers is $\frac{1}{2} \alpha \sum_{k=\varphi+1}^{n} r_{i k}\left(h_{1}+h_{2}\right) h_{1}$. The waiting time cost of the new 
arrival passengers arriving before the arrival of short-turn service is $\frac{1}{2} \alpha \sum_{k=i+1}^{n} r_{i k} h_{2}^{2}$, thus the calculation of passengers waiting time cost $W_{i}^{(2)}$ is obtained as follow:

$$
W_{i}^{(2)}=\frac{1}{2} \alpha \sum_{k=\varphi+1}^{n} r_{i k}\left(h_{1}+h_{2}\right) h_{1}+\frac{1}{2} \alpha \sum_{k=i+1}^{n} r_{i k} h_{2}^{2}, \mu \leq i \leq \varphi-1
$$

In Eq. (16), the calculation of $Y_{i}^{(2)}$ is as follow:

$$
Y_{i}^{(2)}=\rho^{(2)}\left(N_{i}^{(2)}-d\right) l_{i}, \quad i=1,2, \ldots, n-1
$$

In Eq. (17), $N_{i}^{(2)}$ is the cumulative passenger flow from stop $i$ to $i+1$, which can be expressed as:

$$
N_{i}^{(2)}=N_{i-1}^{(2)}+P_{i}^{(2)}-Q_{i}^{(2)}
$$

To sum up, the passenger cost of all-stop scheduling can be expressed by Eq. (19) -

$$
\begin{aligned}
& C^{(2)}=\sum_{i=1}^{\mu-1}\left[\frac{1}{2} \alpha \sum_{k=i+1}^{n} r_{i k}\left(h_{1}+h_{2}\right)^{2}\right]+\sum_{i=\mu}^{\varphi-1}\left[\frac{1}{2} \alpha \sum_{k=\varphi+1}^{n} r_{i k}\left(h_{1}+h_{2}\right) h_{1}+\frac{1}{2} \alpha \sum_{k=i+1}^{n} r_{i k} h_{2}^{2}\right] \\
& +\sum_{i=\varphi}^{n-1}\left[\frac{1}{2} \alpha \sum_{k=i+1}^{n} r_{i k}\left(h_{1}+h_{2}\right)^{2}\right]+\sum_{i=1}^{n-1} \rho_{i}^{(2)}\left(N_{i}^{(2)}-d\right) l_{i} \\
& N_{i}^{(2)}=\left\{\begin{array}{l}
N_{i-1}^{(2)}+\sum_{k=i+1}^{n} r_{i k}\left(h_{1}+h_{2}\right)-\sum_{l=1}^{i-1} r_{l i}\left(h_{1}+h_{2}\right), N_{1}^{(2)}=P_{1}^{(2)}, 1 \leq i \leq \mu-1 \\
N_{\mu-1}^{(2)}+\sum_{k=\varphi+1}^{n} r_{\mu k} h_{1}+\sum_{k=\mu+1}^{n} r_{\mu k} h_{2}-\sum_{l=1}^{\mu-1} r_{l \mu}\left(h_{1}+h_{2}\right), i=\mu \\
N_{i-1}^{(2)}+\sum_{k=\varphi+1}^{n} r_{i k} h_{1}+\sum_{k=i+1}^{n} r_{i k} h_{2}-\sum_{l=1}^{\mu-1} r_{l i}\left(h_{1}+h_{2}\right)-\sum_{l=\mu}^{i-1} r_{l i} h_{2}, \mu<i \leq \varphi-1 \\
N_{\varphi-1}^{(2)}+\sum_{k=\varphi+1}^{n} r_{\varphi k}\left(h_{1}+h_{2}\right)-\sum_{l=1}^{\mu-1} r_{l \varphi}\left(h_{1}+h_{2}\right)-\sum_{l=\mu}^{\varphi-1} r_{l \varphi} h_{2}, i=\varphi \\
N_{i-1}^{(2)}+\sum_{k=i+1}^{n} r_{i k}\left(h_{1}+h_{2}\right)-\sum_{l=1}^{\mu-1} r_{l i}\left(h_{1}+h_{2}\right)-\sum_{l=\mu}^{\varphi-1} r_{l i} h_{2}-\sum_{l=\varphi}^{n-1} r_{l i}\left(h_{1}+h_{2}\right), \varphi<i \leq n-1
\end{array}\right. \\
& \rho_{i}^{(2)}=\left\{\begin{array}{l}
\varepsilon A_{i}^{(2)}, 0 \leq \varepsilon \leq 1, A_{i}^{(2)} \geq A_{0} \\
0, A_{i}^{(2)}<A_{0}
\end{array}, A_{i}^{(2)}=\frac{N_{i}^{(2)}}{b}\right.
\end{aligned}
$$

(2) Bus operation cost

In this paper, the total operating cost of public transit enterprises is divided into two parts, one is the fixed investment cost, the other is operating variable cost.

1) Fixed investment cost

The fixed investment cost of public transit enterprises is related to the minimum fleet size, as shown in Eq. (22)

$$
U=\left(\phi+R+S_{0}\right) u_{\min }
$$


In Eq. (22), $\phi$ is the daily depreciation cost of each vehicle, as shown in Eq. (22); $R$ is the daily wage of drivers on each bus, as shown in Eq. (23);

$$
\begin{gathered}
\phi=\frac{\xi}{\theta \times 365 \times 24} \times T_{r} \\
R=c R_{0}
\end{gathered}
$$

2) Operating variable costs

In the variable cost side, this paper mainly considers the fuel consumption with increase of vehicle mileage. The total fuel consumption can be divided into the fuel consumption of all-stop service $B_{0}$ and that of the short-turn service $B_{1}$. The calculation is shown in Eq. (25) and (26) respectively

$$
\begin{aligned}
& B_{0}=\beta L \\
& B_{1}=\beta L_{s}
\end{aligned}
$$

Thus, the variable cost of bus operation $B$ is expressed as follow:

$$
B=B_{0} f_{0}+B_{1} f_{1}
$$

In Eq. (27), $f_{0}, f_{1}$ is the departure times of all-stop service and short-turn service in the study period respectively.

To sum up, the total system cost calculated by the objective function can be obtained from the sum of passenger costs and operating costs of public transit enterprises in different situations.

When $m=1$, there is one all-stop service participating in the combined dispatching. The objective function of combined dispatching is shown in Eq. (28) - (30). Where, the departure times of all-stop service are equal to the departure times of shortturn service.

$$
\begin{gathered}
\min Z^{(1)}=\omega_{1}\left[C^{(0)} f_{0}+C^{(1)} f_{1}\right]+\omega_{2}\left[B_{0} f_{0}+B_{1} f_{1}+U\right] \\
f_{0}=f_{1}=\frac{\tau}{h_{1}+h_{2}} \\
B_{0}=\beta L, B_{1}=\beta L_{s}
\end{gathered}
$$

Where, $\omega_{1}$ and $\omega_{2}$ are the weight coefficients of relative total cost of public transit enterprises and passengers, and $0<\omega_{1}, \omega_{1}<1, \omega_{1}+\omega_{1}=1$.

When $m=2$, there are two all-stop service participating in the combined dispatching, and the departure times of all-stop service are twice of that of the shortturn service. The objective function of combined dispatching is shown in Eq. (31) (33)

$$
\begin{gathered}
\min Z^{(2)}=\omega_{1}\left[C^{(0)} f_{0}+C^{(1)} f_{1}+C^{(2)} \times \frac{1}{2} f_{0}\right]+\omega_{2}\left[B_{0} f_{0}+B_{1} f_{1}+U\left(u_{\min }\right)\right] \\
f_{0}=2 f_{1}=\frac{2 \tau}{h_{0}+h_{1}+h_{2}}
\end{gathered}
$$




$$
B_{0}=\beta L, B_{1}=\beta L_{s}
$$

Thus, the objective function can be generalized to general situation. When $m$ is any value, the objective value of combined scheduling is shown in Eq. (34) - (36):

$$
\begin{gathered}
\min Z^{(m)}=\omega_{1}\left[C^{(0)} \times \frac{m-1}{m} f_{0}+C^{(1)} f_{1}+C^{(2)} \times \frac{1}{m} f\right]+\omega_{2}\left[B_{0} f_{0}+U\left(u_{\min }\right)\right] \\
f_{0}=m f_{1}=\frac{m \tau}{(m-1) h_{0}+h_{1}+h_{2}}, m=1,2,3, \ldots \\
B_{0}=\beta L, B_{1}=\beta L_{s}
\end{gathered}
$$

When the bus dispatching form is whole vehicle scheduling, the objective function is shown in Eq. (37) - (39):

$$
\begin{gathered}
\min Z^{(1)}=\omega_{1} C^{(0)} f_{0}+\omega_{2}\left[B_{0} f_{0}+U\left(u_{\min }\right)\right] \\
f_{0}=\frac{\tau}{h_{0}} \\
B_{0}=\beta L
\end{gathered}
$$

\subsubsection{Constraints}

In order to meet the needs of passengers and limited by the fleet size, bus companies will set the departure interval according to actual bus line conditions. When the specified maximum and minimum departure intervals are $h_{\max }$ and $h_{\min }$ respectively, it is necessary to ensure that:

$$
\left\{\begin{array}{l}
h_{\min } \leq h_{0} \leq h_{\max } \\
h_{\min } \leq h_{1} \leq h_{\max } \\
h_{\min } \leq h_{2} \leq h_{\max }
\end{array}\right.
$$

\subsection{Model solution}

Analysis of objective function $\min Z^{(m)}$ : the value range of $h_{0}, h_{1}, h_{2}$ is $\left[h_{\min }, h_{\max }\right]$, and under the determined value of $m$, the model solution is to solve a nonlinear optimal problem. Matlab is used to search the total cost to get an optimal solution satisfying $\min Z^{(m)}$, and then the corresponding value of $h_{0}, h_{1}, h_{2}$ under the optimal target value is obtained.

The traditional mathematical method to calculate the optimal solution will lead to too much calculation, which is often unrealistic. Because of its own characteristics, genetic algorithm has a strong formula in solving such problems, so genetic algorithm is used to solve the model [21].

The improvement and function expansion of standard genetic algorithm can be divided into four steps: initialization, fitness calculation, genetic operation to produce offspring, and judgment of termination conditions. 


\subsubsection{Initialization and parameter selection}

- Coding

First, the decision variable is encoded as a binary number, that is, a chromosome $\mathrm{X}$. The relationship between the precision ( $p$ bit after the decimal point) and the encoding length (binary string dimension $q$ ) is as follows:

$$
2^{q-1}<\left(h_{\max }-h_{\min }\right) 10^{p} \leq 2^{q}-1
$$

- Initialization

When determining the initial population, the population size ( $N$ chromosomes) should be determined first. This process is equivalent to randomly selecting $N$ points as the initial solution in the optimization solution space, which is, selecting a set of strings or individuals $X_{i}, i=1,2, \cdots N$.

- Parameter selection

The crossover probability $P_{c}$ and the mutation probability $P_{m}$ are determined.

\subsubsection{Calculation of population fitness}

Since the objective function of the model proposed in this paper is minimal, Eq. (42) is taken as the fitness function:

$$
F(X)= \begin{cases}C_{\max }-f(X), & C_{\max }-f(X)>0 \\ 0, & C_{\max }-f(X) \leq 0\end{cases}
$$

Where $C_{\max }$ is the maximum estimate of $f(X)$.

\subsubsection{Genetic manipulation}

- Selection

In this paper, the Elitist Method and Tournament Selection Method are combined. Where Elitist Method: The individuals with the highest fitness in the population do not carry out pairing exchange, but directly replicate to the next generation; Tournament Selection Method: According to a certain number of individuals randomly selected in the population, the individuals with high fitness are saved to the next generation (in this paper, the tournament scale is selected as 2).

- Crossover

In this paper, the partial matched crossover (PMX) is adopted. PMX operation was proposed by Goldberg and Lingle in 1985. In PMX operation, two exchange points are randomly generated, and the region between the two points is defined as the matching area, and the matching area of two parents is exchanged.

- Mutation

In this algorithm, insertion mutation is used according to probability $P_{m}$. A gene is randomly selected from the chromosome and inserted into other sites at random. 


\subsubsection{Termination conditions}

Before reaching the maximum algebra, judge whether the average fitness value of successive generations of individuals has not changed, or the change value is less than a certain minimum threshold. If so, the iterative process of the algorithm converges and the algorithm ends. Otherwise, replace the previous generation population with the new generation population obtained through selection, crossover and mutation, and return to the selection operation to continue the cycle execution.

\section{Case study}

\subsection{Data acquisition and analysis}

Taking Beijing No. A bus line as an example, there are 24 stops along the up-line. Along the way, there are several subway transfer stations, school surrounding stops and some other crowded areas.

The real-time passenger flow data is collected and analyzed through smart card database. The data collection period is selected as 6:00-22:00. By comparing the passenger flow changes on weekdays and holidays, it is found that there is an obvious imbalance of passenger flow on weekdays. Therefore, it is necessary to optimize the bus scheduling on weekdays. Figure 2 shows the passenger flow variation of bus No. A on weekdays and holidays.

Data statistics: the statistical interval is one hour. The passenger flow data in upward and downward directions of are counted. Taking 6:00-12:00 on weekdays as an example, the passenger flow data is shown in Figure 3.

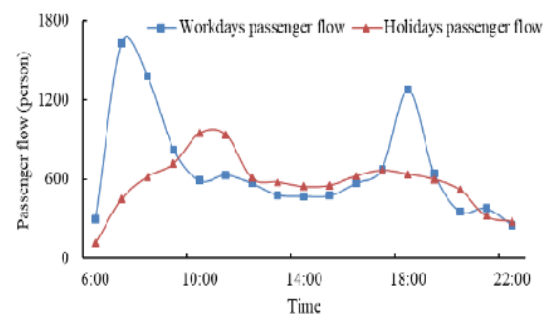

Figure 2. Passenger flow variation of working days and holidays

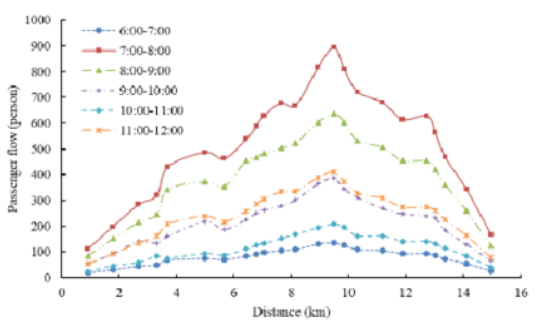

Figure 3. Passenger flow chart

In Figure 3, the ordinate represents passenger flow of the road section, and the abscissa represents the distance between the specific stop and departure stop. It can be seen that the passenger flow in middle section is relatively large, especially during 7:00-8:00 and 8:00-9:00, the passenger flow is relatively concentrated. Therefore, combined with the actual operation situation, it is necessary to optimize the original scheduling scheme by short-turn vehicle combination scheduling. Taking 7:00-8:00 as an example, the calculation results of unbalanced coefficient of road sections are shown in Table 3.

Table 3. Unbalanced coefficient of road section from 7:00 to 8:00

\begin{tabular}{cccccc}
\hline Section & $\begin{array}{c}\text { Passenger } \\
\text { flow }\end{array}$ & $\begin{array}{c}\text { Unbalanced coefficient } \\
\text { of road section }\end{array}$ & Section & $\begin{array}{c}\text { Passenger } \\
\text { flow }\end{array}$ & $\begin{array}{c}\text { Unbalanced coefficient } \\
\text { of road section }\end{array}$ \\
\hline $1-2$ & 112 & 0.204007 & $13-14$ & 836 & 1.522769 \\
$2-3$ & 199 & 0.362477 & $14-15$ & 894 & 1.628415 \\
\hline
\end{tabular}




\begin{tabular}{cccccc}
\hline $3-4$ & 284 & 0.517304 & $15-16$ & 810 & 1.47541 \\
$4-5$ & 320 & 0.582878 & $16-17$ & 711 & 1.295082 \\
$5-6$ & 430 & 0.783242 & $17-18$ & 681 & 1.240437 \\
$6-7$ & 499 & 0.908925 & $18-19$ & 604 & 1.100182 \\
$7-8$ & 452 & 0.823315 & $19-20$ & 601 & 1.094718 \\
$8-9$ & 537 & 0.978142 & $20-21$ & 544 & 0.990893 \\
$9-10$ & 588 & 1.071038 & $21-22$ & 468 & 0.852459 \\
$10-11$ & 629 & 1.145719 & $22-23$ & 344 & 0.626594 \\
$11-12$ & 679 & 1.236794 & $23-24$ & 168 & 0.306011 \\
$12-13$ & 697 & 1.269581 & & & \\
\hline
\end{tabular}

\subsection{Judgment of combined scheduling mode}

Through the statistical analysis of real-time passenger flow, it is found that in 7:00-8:00, 8:00-9:00 and 17:00-18:00, 18:00-19:00, the passenger flow are relatively large and concentrated. After calculating the unbalanced coefficient of road section and conducting on-the-spot investigation, it is found that short-turn service should be set up in these four periods, and the turning back points are stop 11 and 18. In other periods, although some sections meet the judgment conditions, the passenger flow difference is not obvious, the all-stop service dispatching mode is continued. Input parameter setting Through investigation on bus No. A operation group and consulting relevant data, the input parameter values of the model are obtained, as shown in Table 4.

Table 4. Parameter settings

\begin{tabular}{|c|c|c|}
\hline Symbol & Meaning of parameters & Value \\
\hline$\mu$ & Short-turn service departure stop & 11 \\
\hline$\varphi$ & Turning back point of short-turn service & 17 \\
\hline$\tau$ & Duration of model study & $60 \mathrm{~min}$ \\
\hline$L$ & Total length of line & $14.96 \mathrm{~km}$ \\
\hline$L_{s}$ & Length of short-turn service line & $4.12 \mathrm{~km}$ \\
\hline$n$ & Total number of bus stops & 24 \\
\hline$d$ & Number of bus seats & 24 persons/vehicle \\
\hline$b$ & Rated capacity & 90 passengers/vehicle \\
\hline$\alpha_{f}$ & Unit time cost of passengers in off-peak hours & 114 yuan/person $\cdot \min$ \\
\hline$\alpha_{g}$ & Unit time cost of passengers in peak hours & 114 yuan/person $\cdot \min$ \\
\hline$A_{0}$ & Critical load ratio & 1.2 \\
\hline$\varepsilon$ & Parameters of congestion penalty cost coefficient & 0.4 \\
\hline$\beta$ & Unit fuel consumption & 1.42 yuan $/ \mathrm{km}$ \\
\hline$T$ & $\begin{array}{l}\text { Average one-way travel time of all-stop service in peak } \\
\text { hours }\end{array}$ & $61 \mathrm{~min}$ \\
\hline$T_{s}$ & $\begin{array}{l}\text { Average one-way travel time of short-turn service in peak } \\
\text { hours }\end{array}$ & $22 \mathrm{~min}$ \\
\hline$\zeta$ & Bus purchase cost & 300,000 yuan \\
\hline$c$ & Number of drivers per bus & 1 \\
\hline$R_{0}$ & Driver's daily wage & 100 yuan/person d day \\
\hline
\end{tabular}


$S_{0} \quad$ Daily vehicle maintenance fee

$\theta \quad$ Permitted driving years of vehicles

$h_{\text {min }} \quad$ Minimum headway

$h_{\max } \quad$ Maximum headway

$\omega_{1}$ Weight coefficient of passenger cost in total cost

$\omega_{2} \quad$ Weight coefficient of public transit cost in total cost
66.7 yuan/vehicle· day

13 years

$1.5 \mathrm{~min}$

$20 \mathrm{~min}$

0.45

0.55

\subsection{Result analysis}

By solving the single scheduling model and combined scheduling model respectively, the calculation results of the optimal departure interval and frequency under the combined scheduling of all-stop and short-turn are obtained, as shown in Tables 5 and 6.

Table 5. Calculation results of departure frequency and interval under two dispatching modes

\begin{tabular}{|c|c|c|c|c|c|c|}
\hline \multirow[b]{2}{*}{$\begin{array}{c}\text { Time } \\
\text { interval }\end{array}$} & \multicolumn{2}{|c|}{ All-stop fleet scheduling } & \multicolumn{4}{|c|}{ Combined scheduling with short-turn service } \\
\hline & $h_{0}(\min )$ & $\begin{array}{c}f_{0} \\
\text { (vehicle/h) }\end{array}$ & $h_{1}(\min )$ & $h_{2}(\min )$ & $\begin{array}{c}f_{0} \\
\text { (vehicle/h) }\end{array}$ & $\begin{array}{c}f_{1} \\
\text { (vehicle/h) }\end{array}$ \\
\hline $7: 00-8: 00$ & 3.5 & 17.1 & 3.6 & 3.1 & 8.95 & 8.95 \\
\hline $8: 00-9: 00$ & 4.3 & 14.0 & 3.9 & 3.7 & 7.89 & 7.89 \\
\hline $17: 00-18: 00$ & 4.2 & 14.3 & 4.4 & 3.5 & 7.59 & 7.59 \\
\hline $18: 00-19: 00$ & 3.7 & 16.2 & 3.7 & 3.2 & 8.69 & 8.69 \\
\hline
\end{tabular}

Table 6. Calculation results of total departure frequency in different intervals

\begin{tabular}{ccccc}
\hline \multirow{2}{*}{ Time interval } & \multicolumn{2}{c}{ Within short-turn service range } & \multicolumn{2}{c}{ Out of short-turn service range } \\
\cline { 2 - 5 } & $\begin{array}{c}\text { Single } \\
(\text { vehicle/h) }\end{array}$ & $\begin{array}{c}\text { Combination } \\
(\text { vehicle/h) }\end{array}$ & $\begin{array}{c}\text { Single } \\
(\text { vehicle/h) }\end{array}$ & $\begin{array}{c}\text { Combination } \\
\text { (vehicle/h) }\end{array}$ \\
\hline 7:00-8:00 & 17.1 & 17.9 & 17.1 & 8.95 \\
8:00-9:00 & 14.0 & 15.7 & 14.0 & 7.89 \\
17:00-18:00 & 14.3 & 15.2 & 14.3 & 7.59 \\
18:00-19:00 & 16.2 & 17.3 & 16.2 & 8.69 \\
\hline
\end{tabular}

From Table 5 and 6 , it can be found that within the scope of short-turn service section, the departure frequency of combined dispatching mode has been improved, which can better meet the passenger demand of the section with large passenger flow; outside the scope of short-turn service section, the departure frequency of combined dispatching mode is significantly lower than that of the single dispatching mode, which has a positive significance for passenger evacuation and cost saving in peak hours.

Table 7 shows the cost calculation results of single all-stop service dispatching and short-turn service combination dispatching. Meanwhile, Figure 4-6 visually shows the cost comparison before and after the optimization of dispatching mode, in which the total system cost is the sum of passenger cost and bus operation cost.

Table 7. Cost comparison of single all-stop and short-turn service combined scheduling (yuan / h)

\begin{tabular}{ccccccc}
\hline \multirow{2}{*}{ Time interval } & \multicolumn{3}{c}{$7: 00-8: 00$} & \multicolumn{3}{c}{$8: 00-9: 00$} \\
\cline { 2 - 7 } & Single & Combination & Variation & Single & Combination & Variation \\
\hline Passenger cost & 502 & 759 & +257 & 482 & 654 & +172 \\
Operation costs & 1618 & 1246 & -372 & 1484 & 1237 & -247 \\
Total system cost & 2120 & 2005 & -115 & 1966 & 1891 & -75 \\
\hline Time interval & \multicolumn{3}{c}{$17: 00-18: 00$} \\
\hline
\end{tabular}




\begin{tabular}{ccccccc}
\hline & Single & Combination & Variation & Single & Combination & Variation \\
\hline Passenger cost & 461 & 669 & +208 & 437 & 699 & +262 \\
Operation costs & 1602 & 1328 & -274 & 1454 & 1132 & -322 \\
Total system cost & 2063 & 1997 & -66 & 1891 & 1831 & -60 \\
\hline
\end{tabular}

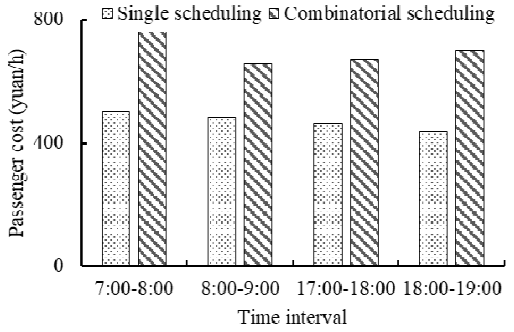

Figure 4. Comparison of passenger cost

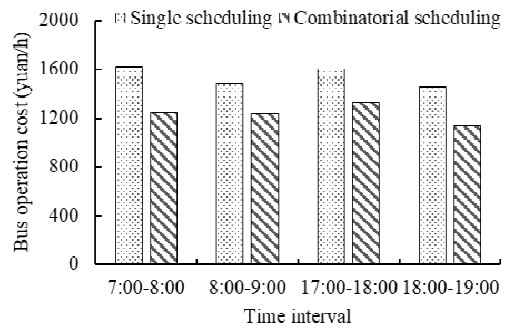

Figure 5. Comparison of bus operation cost

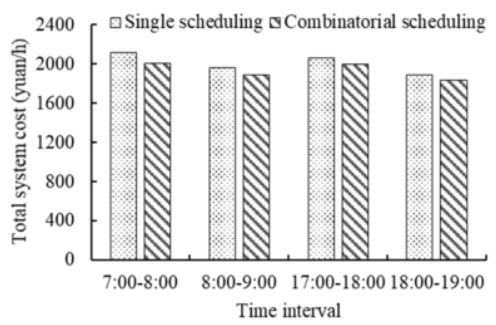

Figure 6. Comparison of total system cost

From Figure 4, Figure 5 and Figure 6, it can be seen that compared with all-stop fleet scheduling, the operation cost of bus companies under combined scheduling scheme is greatly reduced, which can effectively save the total system cost. According to the calculation results of optimal departure frequency and departure interval, the departure interval of other time periods is solved, and the optimal departure interval of each period in whole working day is obtained consequently, as shown in Table 8.

Table 8. Optimal departure interval of each period

\begin{tabular}{|c|c|c|c|c|}
\hline \multirow[b]{2}{*}{ Time interval } & \multirow[b]{2}{*}{ Scheduling } & \multicolumn{3}{|c|}{ Departure interval } \\
\hline & & $h_{0}(\min )$ & $h_{0}(\min )$ & $h_{0}(\min )$ \\
\hline $6: 00-7: 00$ & Single & 11 & - & - \\
\hline \multirow{2}{*}{ 7:00-8:00 } & Single & 3.5 & - & - \\
\hline & Combination & - & 3.6 & 3.1 \\
\hline \multirow{2}{*}{$8: 00-9: 00$} & Single & 4.3 & - & - \\
\hline & Combination & - & 3.9 & 3.7 \\
\hline 9:00-10:00 & Single & 10 & - & - \\
\hline $10: 00-11: 00$ & Single & 8 & - & - \\
\hline $11: 00-12: 00$ & Single & 9 & - & - \\
\hline $12: 00-13: 00$ & Single & 7 & - & - \\
\hline $13: 00-14: 00$ & Single & 10 & - & - \\
\hline $14: 00-15: 00$ & Single & 11 & - & - \\
\hline $15: 00-16: 00$ & Single & 12 & - & - \\
\hline $16: 00-17: 00$ & Single & 9 & - & - \\
\hline \multirow{2}{*}{ 17:00-18:00 } & Single & 4.2 & - & - \\
\hline & Combination & - & 4.4 & 3.5 \\
\hline \multirow{2}{*}{ 18:00-19:00 } & Single & 3.7 & - & - \\
\hline & Combination & - & 3.7 & 3.2 \\
\hline 19:00-20:00 & Single & 7 & - & - \\
\hline $20: 00-21: 00$ & Single & 9 & - & - \\
\hline $21: 00-22: 00$ & Single & 12 & - & - \\
\hline
\end{tabular}


According to the calculation results of optimal departure interval in Table 9, the departure schedule of single dispatching and short-turn combined dispatching can be compiled. In this paper, the smooth transition uniform departure schedule is used to set the departure frequency at the transition of different periods of single scheduling, as shown in Table 9. Figure 7 shows the cumulative departure frequency of 6:00-12:00.

Table 9. Departure frequency of different periods

\begin{tabular}{cccc}
\hline Time interval & Departure frequency $($ vehicle/h) & Time interval & Departure frequency $($ vehicle/h) \\
\hline $6: 00-7: 00$ & 5.45 & $14: 00-15: 00$ & 5.45 \\
7:00-8:00 & 17.13 & $15: 00-16: 00$ & 5.00 \\
$8: 00-9: 00$ & 13.95 & $16: 00-17: 00$ & 6.67 \\
$9: 00-10: 00$ & 6 & $17: 00-18: 00$ & 14.29 \\
$10: 00-11: 00$ & 7.5 & $18: 00-19: 00$ & 16.22 \\
$11: 00-12: 00$ & 6.67 & $19: 00-20: 00$ & 8.57 \\
$12: 00-13: 00$ & 8.57 & $20:-0021: 00$ & 6.67 \\
$13: 00-14: 00$ & 6.00 & $21: 00-22: 00$ & 5.00 \\
\hline
\end{tabular}

Taking the transition section between 6:00-7:00 and 7:00-8:00 as an example, the method for determining departure time is shown in Figure 8. It can be seen from the figure that the departure interval at the transition of two periods is 7:02.

Parameters of genetic algorithm: population size $N=100$; crossover probability $P_{c}=0.95$, mutation probability $P_{m}=0.01$, maximum number of genetic iterations $i=100$, and genetic convergence threshold $\delta=1$.

Finally, the departure time of the bus line is calculated. Previous studies have shown that for the schedule optimization of public transit, the results that are obtained by genetic algorithm are more accurate and reliable[26,27].In order to verify the accuracy of genetic algorithm for solving the proposed model, two widely used heuristic algorithms, simulated annealing algorithm and ant colony algorithm, are compared with the calculation results of genetic algorithm, and it can be found that when the parameters of the three algorithms are within a certain range, the difference between the calculation results is within an acceptable range. In summary, the output of genetic algorithm optimization iteration is taken as the final calculation result. Take 6:00-8:00 as an example, as shown in Table 10. The all-stop scheduling is represented by " 0 " and the short-turn scheduling is represented by " 1 ".

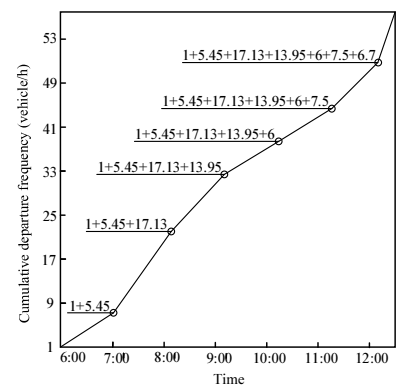

Figure 7. Cumulative departure frequency transition curve of single all-stop dispatching

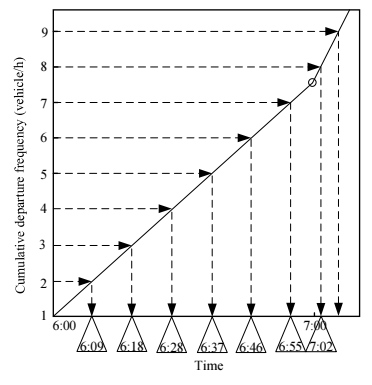

Figure 8. Determination of departure time of section between 6:00-7:00 and 7:00-8:00 
Table 10. Departure schedule during 6:00-8:00 under two different bus dispatching modes

\begin{tabular}{|c|c|c|c|c|c|c|c|c|c|}
\hline \multirow{2}{*}{ No. } & \multicolumn{2}{|c|}{ Single } & \multicolumn{2}{|c|}{ Combination } & \multirow{2}{*}{ No. } & \multicolumn{2}{|c|}{ Single } & \multicolumn{2}{|c|}{ Combination } \\
\hline & $\begin{array}{c}\text { Departure } \\
\text { time }\end{array}$ & Type & $\begin{array}{l}\text { Departure } \\
\text { time }\end{array}$ & Type & & $\begin{array}{l}\text { Departure } \\
\text { time }\end{array}$ & Type & $\begin{array}{l}\text { Departure } \\
\text { time }\end{array}$ & Type \\
\hline 1 & 6:00 AM & 0 & 6:00 AM & 0 & 13 & 7:19 AM & 0 & 7:20 AM & 1 \\
\hline 2 & 6:09 AM & 0 & 6:09 AM & 0 & 14 & 7:23 AM & 0 & 7:23 AM & 0 \\
\hline 3 & $6: 18 \mathrm{AM}$ & 0 & 6:18 AM & 0 & 15 & 7:26 AM & 0 & 7:26 AM & 1 \\
\hline 4 & $6: 28 \mathrm{AM}$ & 0 & $6: 28 \mathrm{AM}$ & 0 & 16 & 7:30 AM & 0 & 7:29 AM & 0 \\
\hline 5 & 6:37 AM & 0 & 6:37 AM & 0 & 17 & 7:33 AM & 0 & 7:33 AM & 1 \\
\hline 6 & $6: 46 \mathrm{AM}$ & 0 & $6: 46 \mathrm{AM}$ & 0 & 18 & 7:37 AM & 0 & 7:36 AM & 0 \\
\hline 7 & $6: 55 \mathrm{AM}$ & 0 & $6: 55 \mathrm{AM}$ & 0 & 19 & 7:40 AM & 0 & 7:40 AM & 1 \\
\hline 8 & 7:02 AM & 0 & 7:02 AM & 0 & 20 & 7:44 AM & 0 & 7:43 AM & 0 \\
\hline 9 & 7:05 AM & 0 & 7:06 AM & 1 & 21 & 7:47 AM & 0 & 7:47 AM & 1 \\
\hline 10 & 7:09 AM & 0 & 7:09 AM & 0 & 22 & 7:51 AM & 0 & 7:50 AM & 0 \\
\hline 11 & 7:12 AM & 0 & 7:13 AM & 1 & 23 & 7:54 AM & 0 & 7:54 AM & 1 \\
\hline 12 & $7: 16 \mathrm{AM}$ & 0 & $7: 16 \mathrm{AM}$ & 0 & 24 & 7:58 AM & 0 & 7:57 AM & 0 \\
\hline
\end{tabular}

By analyzing the two different scheduling schemes, it can be calculated that the running time of short-turn scheduling is $122 \mathrm{~min}$ and that of the all-stop scheduling is 210min. During the peak period 7:00 10:30, the combined dispatching mode has 45 total departure shift, in which 4 short-turn service are dispatched, and the minimum fleet size is 41 . Compared with the all-stop fleet scheduling, the short-turn service combined dispatching reduces the fleet size by $4.9 \%$.

From Eq. (28), the unit vehicle cost per day can be calculated:

$\phi+R+S_{0}=\frac{\xi}{\theta \times 365 \times 24} \times T_{r}+c \cdot R_{0}+S_{0}=\frac{480000 \times 16}{8 \times 365 \times 24}+2 \times \frac{3500}{30}+\frac{2000}{30} \approx 409.5$ (yuan $/$ vehicle $\cdot$ day $)$

According to the calculation results, the combined dispatching mode can save 819 yuan of operation cost every day and effectively improve its economic benefits.

\section{Conclusion and further research}

This paper presents a method to determine the short-turn service combination scheme based on the unbalanced coefficient of passenger flow and a method to determine the section stop. A combinatorial scheduling optimization model is established to minimize the total system cost. The empirical analysis shows that, compared with the single scheduling mode, the optimized short-turn service combination scheduling not only has a better adaptability to peak passenger flow, but also can reduce the departure frequency outside the range of shorting-turning bus section. Our contributions are summarized as follows: (1) On the basis of taking into account the cost of passengers and public transit enterprises, a combined scheduling model of short-turning service is established with considering different scheduling strategies, and the solution algorithm is designed. (2) A framework of designing bus combination scheduling scheme based on real-time data is proposed, and the processing method of obtaining passenger flow data based on bus smart card is studied, which can determine the short-turn point more accurately and present the practical instructions to transit operators. (3) A case study of the No. A bus line in Beijing for the service time of a whole day is carried out, the analysis results show that the short-turning service is necessary for the peak hours in working day. By compared with the actual operation, the departure scheme and total 
cost are presented, which shows the feasibility and effectiveness of our proposed method.

Although the proposed combined dispatching model can effectively solve the problems existing in the public transit dispatching, the scope of public transport dispatching is relatively wide. In terms of model generalization, it is necessary to evaluate the model under different parameters by adjusting the value of variables according to actual situation in different regions. In addition, some limitations in this paper need further study: (1) Only the peak passenger flow of a single bus line is analyzed in this paper. However, the research scope can be expanded to further study the regional dispatching optimization. (2) This paper does not design other scheduling forms, such as the combined scheduling of all-stop service, short-turn service and stopskipped bus. The impact of more complex scheduling forms on the cost of passengers and bus companies is worth further consideration. (3) Last but not least, only static combined scheduling scheme is obtained, and the intelligent dynamic combined scheduling model and the more efficiently solution algorithm should be studied in the future.

\section{Acknowledgments}

The authors would like to express their sincere gratitude for the funding support provided by the National Key R\&D Program of China (Grant No.2018YFB1601200).

\section{References}

[1] Cancela H, Mautton A, Urquhart ME. Mathematical programming formulations for transit network design. Transportation Research Part B: Methodological. 2015 Jul; 77:17-37.

[2] Cancela H, Mautton A, Urquhart ME. Mathematical programming formulations for transit network design. Transportation Research Part B: Methodological. 2015 Jul; 77:17-37.

[3] Liu T, Ceder A. Deficit function related to public transit: 50 year retrospective, new developments, and prospects. Transportation Research Part B: Methodological. 2017 Jun; 100:1-19.

[4] Deng LB, Gao W, Zhou WL, et al. Optimal Design of Feeder-bus Network Related to Urban Rail Line based on Transfer System. 2013, 96:2383-2394.

[5] Wenxin Li, Qiyuan Peng, Qinlin Li, et al. Joint Operating Revenue and Passenger Travel Cost Optimization in Urban Rail Transit. 2018, 2018:1-15

[6] Tomáš R, Shadi SA, Yousef $M$ et al. Train timetable design under elastic passenger demand. Transportation Research Part B. 2018, 111

[7] Peña D, Tchernykh A, Nesmachnow S, Massobrio R, Feokitistov A, Bychkov I, et al. Operating cost and quality of service optimization for multi-vehicle-type timetabling for urban bus systems. Journal of Parallel and Distributed Computing. 2019 Nov; 133:272-85.

[8] Liu T, Ceder A, Chowdhury S. Integrated public transport timetable synchronization with vehicle scheduling. Transportmetrica A: Transport Science. 2017 Jul; 13(10):932-54.

[9] AlKheder S, AlRukaibi F, Zaqzouq A. Optimal bus frequency for Kuwait public transit company: A cost view. 2018 Aug; 41:312-9.

[10] Giesen R, Martínez H, Mauttone A, Urquhart ME, et al. A method for solving the multi-objective transit frequency optimization problem. Journal of Advanced Transportation. 2017 Apr; 50(8):2323-37.

[11] Berrebi SJ, Watkins KE, Laval JA. A real-time bus dispatching policy to minimize passenger wait on a high frequency route. Transportation Research Part B: Methodological. 2015 Nov; 81(2):377-89.

[12] Park C, Lee J, Sohn SY. Recommendation of feeder bus routes using neural network embedding-based optimization. Transportation Research Part A: Policy and Practice. 2019 Aug; 126:329-41.

[13] John J, Bartholdi, et al. A self-coördinating bus route to resist bus bunching. Transportation Research Part B Methodological, 2012 Oct;46(4):481-91 
[14] Canca D, Barrena E, Laporte G, et al. A short-turning policy for the management of demand disruptions in rapid transit systems. Annals of Operations Research. 2016 Jul; 246(1-2):145-66.

[15] Cheng SJ. Research on short-turn vehicle combinational scheduling method for bus route. Huazhong University of Science and Technology. 2013.

[16] Cortés CE, Jara-Díaz S, Tirachini A. Integration short turning and deadheading in the optimization of transit services. Transportation Research Part A: Policy and Practice. 2011 Jun; 45(5): 419-34.

[17] Hu BY, Wang XK, Chen WQ. Study on combinational scheduling between inter-zone vehicle and regular vehicle for urban public transit. Journal of Wuhan University of Technology (Transportation Science \& Engineering). 2012 Dec; 36(6):1192-95.

[18] Cao YL. Study on the optimal model and solution for the problem of public transport dispatch. Experiment Science and Technology 2011 Aug;9(4):27-30,182

[19] Chen JX, Liu ZY, Zhu SL, Wang W. Design of limited-stop bus service with capacity constraint and stochastic travel time. Transportation Research Part E: Logistics and Transportation Review. 2015 Nov; 83:1-15.

[20] Wei M, Chen XW, Sun B. Multi-objective single line transit mixed scheduling model considering express bus service. Journal of Transportation Systems Engineering and Information Technology. 2015 Apr; 15(2):169-74,181.

[21] Canca D, Barrena E, Laporte G, Ortega FA. A short-turning policy for the management of demand disruptions in rapid transit systems. Annals of Operations Research. 2014 Jul; 246(1-2): 145-66.

[22] Zhang H, Zhao SZ, Cao Y, Liu HS, Liang SD. Real-time integrated limited-stop and short-turn service control with stochastic travel time. Journal of advanced transportation. 2017 Jul; 2017(746):1-9.

[23] Gao L. Research and application of vehicle bus combined scheduling model for urban public interval bus. Dalian Maritime University. 2017.

[24] Ming J, Zhang GJ, Liu YD. Combinatorial optimization model of multi-modal transit scheduling. Computer Science. 2015 Sept; 42(9):263-7.

[25] Liu C. Research on single line bus scheduling problem based on hybrid heuristic algorithm. Beijing Jiaotong University. 2016.

[26] Huang AL, Dou ZQ, Qi LZ, Wang LW. Flexible Route Optimization for Demand-Responsive Public Transit Service. Journal of Transportation Engineering, Part A: Systems, 2020.Sep;146(12): 04020132

[27] Xiong, J, He Z, Guan W, Ran B. Optimal timetable development for community shuttle network with metro stations. Transportation Research Part C: Emerging.2015 Nov; 60: 540-565. 